\title{
Constraint-free Topological Mapping and Path Planning by Maxima Detection of the Kernel Spatial Clearance Density
}

\author{
Panagiotis Papadakis, Mario Gianni, Matia Pizzoli, Fiora Pirri \\ ALCOR Vision Perception and Cognitive Robotics Laboratory \\ Department of Computer and System Sciences, University of Rome "La Sapienza”, Italy \\ \{papadakis, gianni.mario, pizzoli, pirri\}@dis.uniromal.it
}

Keywords: $\quad$ topological mapping; path planning; mean shift; distance transform

\begin{abstract}
Asserting the inherent topology of the environment perceived by a robot is a key prerequisite of high-level decision making. This is achieved through the construction of a concise representation of the environment that endows a robot with the ability to operate in a coarse-to-fine strategy. In this paper, we propose a novel topological segmentation method of generic metric maps operating concurrently as a path-planning algorithm. First, we apply a Gaussian Distance Transform on the map that weighs points belonging to free space according to the proximity of the surrounding free area in a noise resilient mode. We define a region as the set of all the points that locally converge to a common point of maximum space clearance and employ a weighed meanshift gradient ascent onto the kernel space clearance density in order to detect the maxima that characterize the regions. The spatial intra-connectivity of each cluster is ensured by allowing only for linearly unobstructed mean-shifts which in parallel serves as a path-planning algorithm by concatenating the consecutive mean-shift vectors of the convergence paths. Experiments on structured and unstructured environments demonstrate the effectiveness and potential of the proposed approach.
\end{abstract}

\section{INTRODUCTION}

In real-world robotic applications where environmental constraints are minimal, formulating the perceived knowledge acquired from incoming sensory data into meaningful, higher-level representations of the environment, is a prerequisite of high-level decision making (Murphy, 2004; Maxwell et al., 2004). Among the various robotic sensors, mobile robots are commonly equipped with range scanners that allow for mapping of the environment and navigating within the created map. By modelling a map as an occupancy grid map (either 2D or 3D), a robot can be initially endowed with a set of basic functionalities ranging from obstacle avoidance and low-level path planning to frontier-based exploration.

An important prerequisite for the development of higher-level functionalities is a representation of the environment that captures the topological structure of the free area. This is the result of a topological segmentation of the metric map, a process guided by the morphological features that determine the distinction between regions. By building a graph structure of the environment where nodes correspond to topological regions and edges determine the traversability be- tween adjacent regions, a robot is able to operate in a coarse-to-fine strategy (Kuipers et al., 2004), (Eppstein et al., 2006),(Gianni et al., 2011) using a hierarchical arrangement of the perceived knowledge about the environment.

In this paper, we propose a novel method for topologically segmenting metric maps acquired by mobile robots in order to concisely capture the spatial characteristics of the environment that are involved in higher-level decision making. The input to the proposed method is an occupancy grid wherein each grid cell corresponds to space that is designated as free, occupied or unknown. Merging the latter two cases, we obtain a boolean occupancy grid that is provided as input to a Gaussian Euclidean Distance Transformation (GEDT). By setting a threshold on the GEDT of the map we classify the free areas into those that are safely accessible by a robot in terms of distance from surrounding obstacles. In the sequel, we cluster these areas by detecting the local maxima of the GEDT via a weighed mean-shift (Comaniciu and Meer, 2002; Fukunaga and Hostetler, 1975) gradient ascent, where cells are weighed by the corresponding GEDT value. Each cluster is formed by all the points that locally converge to the same point of maximum GEDT value, 
i.e. maximum space clearance. To prevent from clustering together cells that are spatially disjoint, we constrain the mean-shift vectors by allowing only for linearly unobstructed line segments. This ensures that cells in the same cluster are actually reachable by the robot by using the convergence paths of the meanshift clustering process that is in parallel functioning as a path planning algorithm.

The proposed formulation resembles the intuitive process for topological segmenting the free space in the absence of prior knowledge about the environment, i.e. a topological region comprises of all the points that locally converge to a common point of maximum clearance-openness of free space where the convergence path is composed of steps only within free space.

The contributions of the present work are summarized as follows:

- We propose a novel topological mapping method concurrently operating as a path planning algorithm.

- The proposed topological mapping method is robust to small topological changes, resilient to noise and is applicable to structured as well as unstructured-outdoor environments.

- The proposed path planning method, implicitly derived from the proposed topological mapping framework, yields the optimal paths for a robot in terms of safety distance from surrounding obstacles and maximum peripheral visibility.

The remainder of this paper is organized as follows: In Section 2, we briefly recall the state-of-theart in topological mapping methods identifying the respective prominent streams of research. In Section 3, we describe in detail the framework in which we compute the GEDT of a map and employ an adapted mean-shift gradient ascent for clustering the free space and topologically segmenting the metrical map. In Section 4, we evaluate the proposed method through experiments in structured and semistructured environments that demonstrate its feasibility and effectiveness and finally, in Section 5, we summarize the contributions of this paper.

\section{RELATED WORK}

Topological maps capture the structure of the perceived environment in a concise representation that allows high-level reasoning and planning together with an easier human-robot interaction. In comparison to metric maps, topological maps are more com- pact and less sensitive to errors that are accumulated due to noisy sensor readings.

Depending on the sensors that are used for perceiving the environment, we could distinguish topological mapping methods into those that use visual sensory data from cameras (appearance-based) and those that use geometric sensory data from range scanners (geometry-based).

Appearance-based methods employ scene understanding and object detection in order to derive the semantics of regions within the explored environment (Goedeme et al., 2007),(Goedeme et al., 2008),(Liu et al., 2009),(Montijano and Sagues, 2011),(Murillo et al., 2007),(Valgren and Lilienthal, 2008),(Murillo et al., 2009),(Paul and Newman, 2010) and build a topological graph wherein nodes are assigned to detected landmarks. Creating a topological graph of the environment is imperative in order to efficiently process and maintain the huge amount of information that is acquired from the cameras.

In this paper, we focus on the second category of topological mapping methods that are based on the morphological features of the shape of the environment as perceived by range sensors such as lasers. In structured-indoor environments, the most common feature that is used regards the distinction of free space into large open spaces that are interconnected through narrow passages or gateways. These passages determine the borders among distinct regions that comprise the topological map.

Within this spirit, a number of relevant works can be identified. In the pioneering work of Thrun (Thrun, 1998) the borders of the topological regions were determined by finding the critical points within the Voronoi diagram of the free space that denote the points where space clearance is locally minimized, a concept that was also initially adopted by Beeson et al. in (Beeson et al., 2003). In their following work (Beeson et al., 2005), they proposed the Extended Voronoi Graph defined as the subset of the Generalized Voronoi Graph (GVG) that is closer than a fixed threshold from any obstacle, added to the set of all equidistant points from the closest obstacle. Myung et al. (Myung et al., 2009) extract the GVG of an eroded metric map in order to detect the position of the narrowest passages while Joo et al. (Joo et al., 2010) compute the curvature of the map border to detect candidate cutting points. In the work of Fabrizi et al. (Fabrizi and Saffiotti, 2000; Fabrizi and Saffiotti, 2002), concepts of digital topology are used as the basis of the closure and opening mathematical morphological operators that are applied in fuzzy gridmaps producing constrained regions along with geometric information using moments. 
Spectral clustering has been widely used for topological segmentation of metric maps as an approximate solution to the normalized cut of a graph (Shi and Malik, 2000). In the work of Brunskill et al. (Brunskill et al., 2007), the initial set of graph nodes is constructed by random sampling of the free space and edges are created depending on the visibility between the nodes while the method proposed by Choi et al. (Choi et al., 2009) is initially driven by a quadtree cell decomposition of free space. To support incremental construction of the topological graph during exploration the former detect discontinuities between the eigenvalues of the similarity matrix, while the latter measure the convexity of the regions. Zivkovic et al. (Zivkovic et al., 2006) employed spectral clustering at the finest level of resolution of the occupancy grid and determine the optimal number of clusters by minimizing a cost function that relies on the structure of the eigenvectors of the Laplacian. In recent work, Liu et al. (Liu et al., 2011) highlighted limitations in spectral clustering based topological segmentation related to its instability under varying settings of parameters and map examples. In their approach they compute the mutual information graph of the occupancy grid and segment it using Chow-Liu tree factorization.

While a study of the current approaches provides a thorough understanding of the topological mapping problem, it appears that the majority of methods is formulated and evaluated in structured-indoor environments where the segmentation process is driven by the presence of rooms, corridors, doors, etc. On the contrary, in applications of minimal environmental constraints, such as Urban Search and Rescue, an explicit underlying structure of the explored environment is unlikely to exist. In addition, the noise induced from the sensors becomes more pronounced as a result of operating under a broader range of environmental conditions such as the presence non-reflecting surfaces, smoke or fire, etc. In the next section we unfold the proposed topological segmentation method that is accordingly formalized to be robust to noise and relatively small changes of the environment.

\section{TOPOLOGICAL MAPPING}

In this Section we describe the method that we propose to segment a generic metrical map given in the form of a boolean occupancy grid, into a set of topological regions. The proposed segmentation method adheres to the following criteria:

i. A topological region is identified by a point of maximum space clearance, i.e. whose distance from surrounding obstacles is locally maximized. ii. All points that locally converge to the same point of maximum space clearance belong to the same region.

iii. The convergence path of a point to the point of locally maximum clearance comprises only of steps within free space.

\subsection{Distance-based classification of free space}

The first criterion formulates a space segmentation approach that is beneficial for a mobile robot in terms of safety and visibility. With respect to safety, a point in space that has locally the furthest distance from surrounding obstacles corresponds to a position that the robot can efficiently reach by following a path with reduced obstacle interference. In terms of visibility, a point of maximum space clearance corresponds to the position where occlusions are minimized and a robot can have a broad overview of the surrounding area. Furthermore, this criterion resembles the intuitive human process of space segmentation into rooms and areas in general. Whenever we stand in a position very close to occupied space where visibility is relatively constrained we do not identify the center of a region, rather, when we stand in the center of a region we commonly have a relatively large distance from obstacles and a broad, clear view of the surrounding area.

To obtain a space classification of the metric map according to the distance from surrounding obstacles, the proposed approach adheres to the Distance Transform.

More formally, we denote a boolean occupancy grid of cells as a function $C: \mathbf{p} \rightarrow\{0,1\}$ where $\mathbf{p}=$ $\left\{\mathbf{p}_{i}\left(x_{i}, y_{i}\right) \mid i=1,2, \ldots, n\right\}$ is the set of cells and $n$ is the cardinality of the set. Letting $F=\{\mathbf{p} \mid C(\mathbf{p})=0\}$ denote the set of free cells and $O=\{\mathbf{p} \mid C(\mathbf{p})=1\}$ the set of occupied cells, the Euclidean Distance Transform $D_{f}(\mathbf{p})$ of the free area is given by:

$$
D_{f}(\mathbf{p})=\min _{\mathbf{p}_{k} \in O}\left\|\mathbf{p}-\mathbf{p}_{k}\right\|
$$

where $\|$.$\| denotes the Euclidean distance.$

Ideally, the distance transform would be sufficient to obtain a classification of the points in terms of space clearance as shown in Figure. 1 (b) for the simulated environment shown in Figure. 1 (a). In practice however, metric maps obtained from robotic sensors are highly susceptible to noise as shown for a typical example in Figure. 1 (c) together with the corresponding EDT image in Figure. 1 (d). Due to noisy measurements, individual grid cells that are designated as occupied may affect the EDT values of all the neighbouring cells. This results in an undesirable reduction 


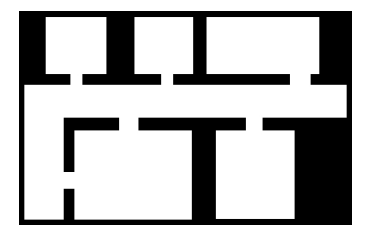

(a)

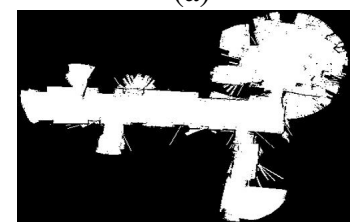

(c)

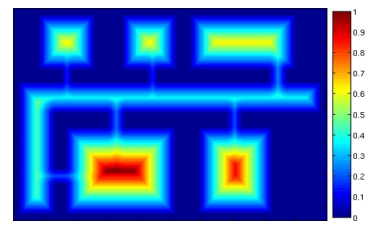

(b)

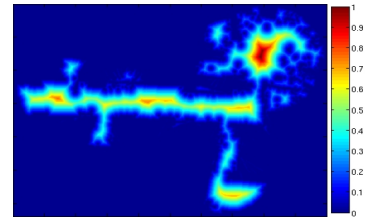

(d)
Figure 1: Example occupancy grids (a),(c) and the corresponding Euclidean Distance Transforms (b),(d).

of the estimated space clearance in relatively large open areas.

To decrease the sensitivity to noise and to relatively small obstacles, we compute the discrete convolution of $C$ with the Gaussian kernel $G(\mathbf{p})=$ $\frac{1}{2 \pi \sigma^{2}} \exp \left(-\frac{\|\mathbf{p}\|^{2}}{2 \sigma^{2}}\right)$ given by:

$$
\widetilde{C}(\mathbf{p})=\sum_{i=1}^{n} C\left(\mathbf{p}_{i}\right) G\left(\mathbf{p}-\mathbf{p}_{i}\right)
$$

According to this formulation we derive that $\tilde{C}(\mathbf{p}) \in$ $[0,1)$ hence for free cells lying close to occupied space $\tilde{C}(\mathbf{p}) \rightarrow 1$ while for free cells lying away from occupied space $\tilde{C}(\mathbf{p}) \rightarrow 0$. For the purpose of the implementation that is followed in this paper (as detailed in Section 3.2), we encode this information by setting $\widetilde{D}(\mathbf{p})=1-\widetilde{C}(\mathbf{p})$ so that cells lying away from obstacles will be characterized by a higher value than the cells lying close to obstacles. The inversion is purposely adopted after computing the convolution since the computation of the latter is more efficient when sparse matrices are used (in practise we compute the convolution using a gaussian kernel of fixed size containing $99 \%$ of the energy). In Figure 2 (a) we show the initial grid map and in Figure 2 (b) the corresponding $\tilde{D}$.

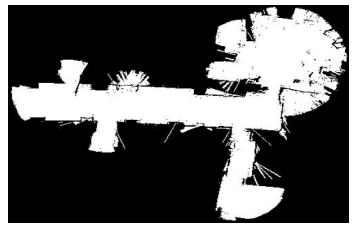

(a)

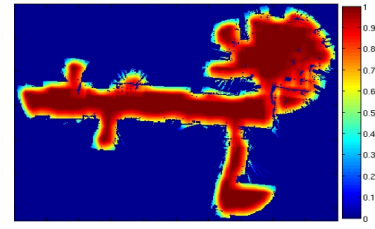

(b)
Figure 2: Example noisy occupancy grid (a) and the corresponding Gaussian Euclidean Distance Transform (b).

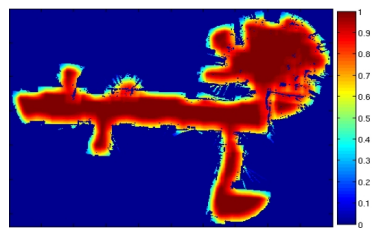

(a)

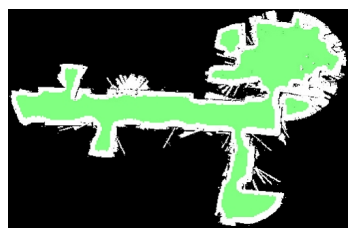

(b)
Figure 3: Determination of safely accessible free area (shown in green) (b) by setting a threshold on the distance from surrounding obstacles (a).

In comparison to the corresponding EDT shown in Figure 1 (d) the convolved occupancy grid is more robust to noise and relatively small obstacles which results in an amended classification of free space with respect to the distance from surrounding obstacles. In the following, we apply a threshold $t_{s}$ in $\widetilde{D}$ to signify the areas that are considered as safely accessible by a mobile robot, in terms of distance from surrounding obstacles. The value of the threshold depends on the size of the footprint-radius of the mobile robot as well as the confidence to its estimated localization within the map. In Figure 3 we show the remaining free area as a result of setting a threshold to $\tilde{D}$.

The magnitude of the $\sigma$ of the kernel determines the importance that is given to surrounding obstacles in terms of their distance from a particular free cell. By decreasing $\sigma$ we give more confidence to the immediate close neighbourhood of the free cell while by increasing $\sigma$ we can cover bigger areas around the cell and consider obstacles that are more distant. The determination of a fixed value is a balance between these aspects and is related to the resolution of the grid map.

\subsection{Weighed Mean-shift clustering of free space}

The next step is to detect the local maxima within $\widetilde{D}$, i.e. the points of maximum space clearance that will represent the centres of the topological regions, together with all the points that belong to the same region. Towards this goal, we employ a mean-shift (Comaniciu and Meer, 2002) gradient ascent on the $\tilde{D}$ image of the occupancy grid, adapted to conform to the criteria (as described in Section 3) on which the proposed segmentation process is based. In practise, depending on the size of the occupancy grid, a downsampled version of $\tilde{D}$ may be preferred in order to reduce the overall computational cost.

In the following, we give the preliminary technical details of mean-shift clustering (for the detailed formulation see (Comaniciu and Meer, 2002)) needed 
to make the paper self contained in accordance to the notations already used.

Let $u=\left\{i \mid \mathbf{p}_{i} \in F\right\}$ then given a cell $\mathbf{p}$ that lies in free space, its kernel density estimate $f_{k}$ is given by:

$$
f_{k}(\mathbf{p})=\frac{1}{n b^{2}} \sum_{u} k\left(\frac{\left\|\mathbf{p}-\mathbf{p}_{u}\right\|^{2}}{b^{2}}\right)
$$

where $b$ is the bandwidth of a radially symmetric kernel and $k$ the corresponding profile function.

By setting $g(x)=-k^{\prime}(x)$ and taking the gradient $\nabla$ of eq. (3) we obtain:

$$
\mathbf{m}_{s}(\mathbf{p})=\frac{\sum_{u} \mathbf{p}_{u} g\left(\frac{\left\|\mathbf{p}-\mathbf{p}_{u}\right\|^{2}}{b^{2}}\right)}{\sum_{u} g\left(\frac{\left\|\mathbf{p}-\mathbf{p}_{u}\right\|^{2}}{b^{2}}\right)}-\mathbf{p}
$$

that denotes the mean-shift vector which points towards the direction of maximum increase of the kernel density. By repeatedly applying the mean-shift operator, we can identify the points of locally maximum space clearance as the points where the gradient of eq. (3) is minimized, according to a convergence threshold $\varepsilon$.

For the computation of the kernel density we employ the Epanechnikov kernel which minimizes the square error between the density and its estimate (Comaniciu and Meer, 2002). The profile $k_{E}$ of the Epanechnikov kernel is given by:

$$
k_{E}(x)= \begin{cases}1-x, & \text { if } 0 \leq x \leq 1 \\ 0, & \text { if } x>1\end{cases}
$$

We adapt the former formulation of mean-shift to consider not only the spatial position of a data point in the estimation of the kernel density, but also its contribution as specified by a weight factor. This factor is the corresponding $\widetilde{D}(\mathbf{p})$ value that determines the space clearance of the point. Hence, we compute the modified shift vector as:

$$
\mathbf{m}_{s}(\mathbf{p})=\frac{\sum_{u} \mathbf{p}_{u} g\left(\frac{\left\|\tilde{D}(\mathbf{p}) \cdot \mathbf{p}-\tilde{D}\left(\mathbf{p}_{u}\right) \cdot \mathbf{p}_{u}\right\|^{2}}{b^{2}}\right)}{\sum_{u} g\left(\frac{\left\|\tilde{D}(\mathbf{p}) \cdot \mathbf{p}-\tilde{D}\left(\mathbf{p}_{u}\right) \cdot \mathbf{p}_{u}\right\|^{2}}{b^{2}}\right)}-\mathbf{p}
$$

The convergence to the modes of the density produces slightly different locations due to the threshold $\varepsilon$ that determines the termination of the mean-shift iterations, as well as because the value of the gradient is very close to zero within regions where the density estimate is constant. These artifacts are eliminated by fusing mode candidates at a distance less than the kernel bandwidth $b$ by choosing the one corresponding to the highest density.

The magnitude of the bandwidth $b$ of the kernel sets the size of the window that is considered for the computation of the mean-shift and therefore determines the surface area of the topological regions. The bigger bandwidth we select, the bigger areas are detected and vice versa.

\subsection{Constraining of mean-shifts to linearly unobstructed vectors}

According to the former formulation of mean-shift, there are no constrains in the computation of the $\mathbf{m}_{s}$ vector. This may result in computing mean-shift vectors that point to positions that do not belong to free space or that comprise of cells lying within occupied space. Hence, a cell may converge to a point of locally maximum space clearance although the two positions are spatially disjoint. In Figure 4 (a) we show a typical example of this case.

In the case of topological map segmentation, we require that every point that belongs to a topological region is spatially accessible by a robot through a path whenever the robot is located within this region. To avoid the costly computation of paths within the metric map, we introduce this condition into the convergence path of the mean-shift clustering procedure. In detail, for each cell we condition the mean-shift vectors of the corresponding convergence path to allow only linearly unobstructed vectors. In this way, we prevent the mean-shift vector from jumping into or crossing through occupied space and the convergence path simulates a path that could be followed by the robot in real.

In order to ensure the spatial intra-connectivity of cells, we use Bresenham's line algorithm (Theoharis et al., 2008) through which we can recover the cells that lie along each mean-shift vector. If for a particular mean-shift vector all the corresponding cells belong to free space, then mean-shift proceeds normally to the next iteration. Otherwise, we decrease the bandwidth to $b^{\prime}=b-1$ and recompute the meanshift vector. This is repeated until all cells along the mean-shift vector lie on free space wherein convergence advances or until $b^{\prime}=1$ where we identify an isolated free cell that is discarded. The consistent clustering of the example shown in Figure 4 (a) is given in Figure 4 (b).

The advantage of the former formulation of meanshift relies on the usability of the convergence paths of all cells to the corresponding centres of the topological regions for the purpose of low-level path planning. In particular, by concatenation of the consecutive mean-shift vectors for each cell we obtain the set of convergence paths that a robot can readily follow in order to navigate to the center of its topological region. By repeated concatenation of the conver- 


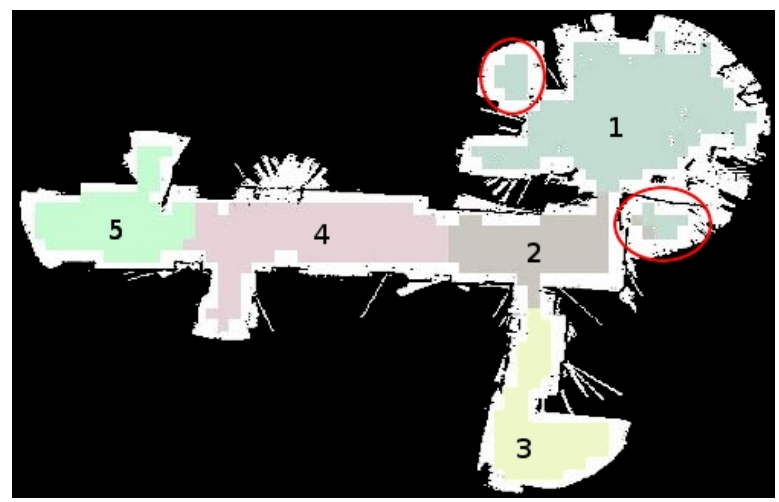

(a)

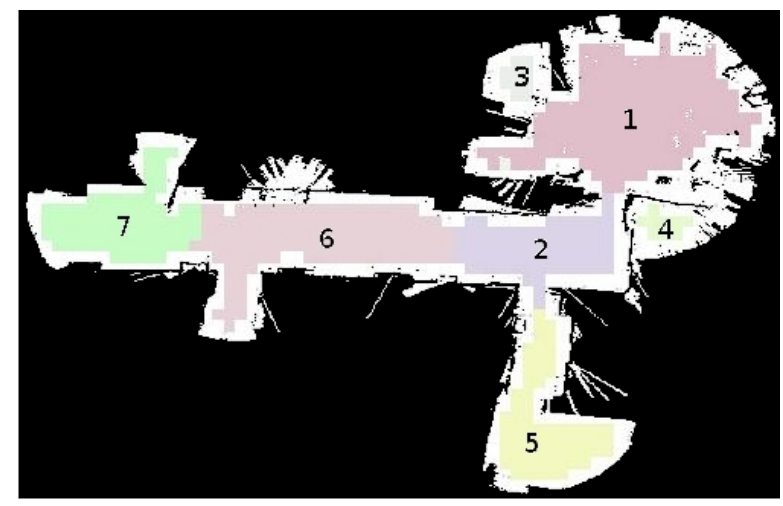

(b)

Figure 4: (a) Example of clustering together spatially disconnected areas. Topological regions are identified by the index of their cluster and their corresponding color. The cells belonging to the areas highlighted in red are erroneously incorporated in region \#1 or \#2 although they are spatially disjoint; (b) Example of consistent clustering by constraining mean-shifts to allow only for linearly unobstructed vectors by using Bresenham's line algorithm in order to ensure the intra-connectivity of cells.

gence paths of adjacent cells that belong to different regions, longer paths can be constructed that endow a robot to navigate to any position within the map by following a path that passes through the centres of the intermediate topological regions. These paths are optimal with respect to the safety of the robot in terms of overall distance from surrounding obstacles as well as visibility in terms of space clearance. In Figure 5 we show the convergence paths of cells to the points of locally maximum space clearance.

\section{EXPERIMENTS}

In this Section we present the experiments that demonstrate the feasibility and effectiveness of the proposed topological segmentation method.

We divide our experiments in two parts. First we analyse the role of the parameters involved in the pro-

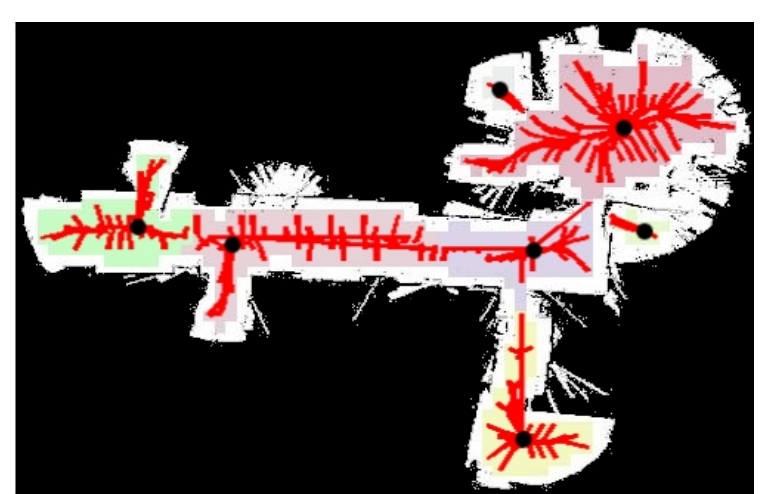

Figure 5: Convergence paths of a subset of grid cells (shown in red) to the corresponding points of locally maximum space clearance (shown in black). posed methodology and second we provide a qualitative evaluation of the proposed topological segmentation in a variety of metric maps including structured, semi-structured and totally unstructured maps.

The parameters of the proposed segmentation method are set by taking into account the characteristics of the robot and its sensors. The values that need to be set concern the variance $\sigma$ of the Gaussian function used for the convolution (eq. 2), the threshold $t_{s}$ for the determination of the safely accessible areas and the bandwidth parameter $b$ for the estimation of the kernel density (eq. 3, 4).

The $\sigma$ parameter determines the importance of surrounding obstacles according to their distance from a particular free cell. By decreasing $\sigma$, i.e. the uncertainty, we increase the confidence to the immediate close neighbourhood of the free cell. This increases the ratio of cells that are designated as having maximum space clearance to all the other cells that lie closer to occupied space. In other words, relatively small free areas are characterized by high space clearance. By increasing $\sigma$ bigger areas around a cell are covered by considering space that is more distant which decreases the aforementioned ratio. Hence, only wide open areas are characterized by having high space clearance. The effect of various $\sigma$ values $(\sigma=15,30,45)$ in the estimation of space clearance is demonstrated in Figure 6.

The threshold $t_{s}$ is set in proportion to the size of the robot, since the bigger the robot the further it should reside from occupied space and vice versa. By increasing $t_{s}$, the free area with sufficiently high space clearance is decreased hence also decreasing the area to be clustered. This may isolate portions of free space if the access is through relatively narrow passages and hence not traversable by the robot. 


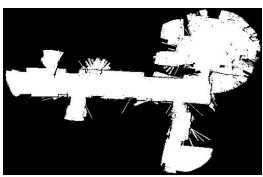

(a)

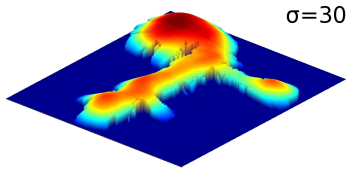

(c)

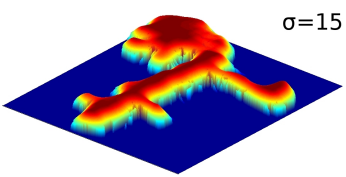

(b)

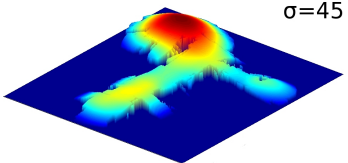

(d)
Figure 6: (a) Example metric map $(499 \times 319)$ and (b)-(d) the estimated space clearance for varying $\sigma$.

In Figure 7 we show the effect of different thresholds $\left(t_{s}=0.8,0.9,0.99\right)$ in estimating the safely accessible free space by a robot.

The bandwidth parameter $b$ related to the estimation of the kernel space clearance density determines the surface area of the segmented regions. It defines the radius of the kernel window, i.e. the neighbourhood around a cell in the estimation of the mean-shift vector. By increasing the radius we allow for longer mean-shift vectors and thus clustering together cells that are more distant from one another which typically results in less number of clusters. Setting a particular value for the bandwidth depends on the desired granularity of the segmentation process that can vary in order to construct either coarse or fine topological maps. In Figure 8 we show the effect of different bandwidth values $(b=3,5,7)$ in the final clustering.

In Figure 9 we demonstrate the stability of the proposed segmentation method in the presence of noise or relatively small obstacles within a given map. It is fairly evident that the effect of noise or very small obstacles in the clustering result is minimal as relatively

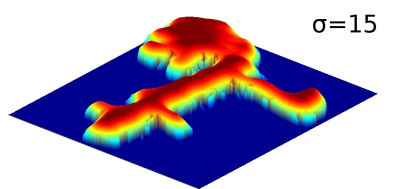

(a)

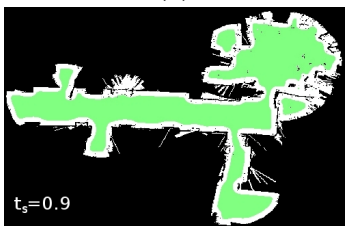

(c)

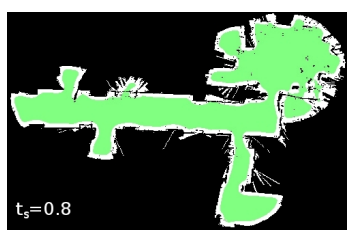

(b)

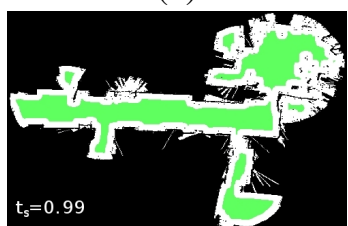

(d)
Figure 7: (a) Space clearance estimation of metric map and (b)-(d) determination of safely accessible areas for a robot (in green) using different thresholds.

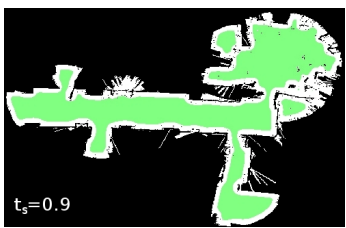

(a)

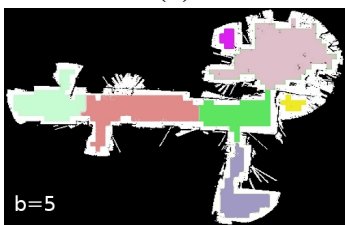

(c)

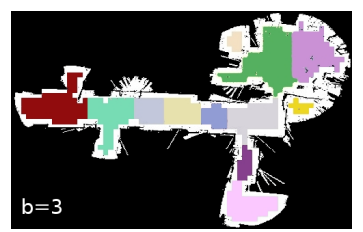

(b)

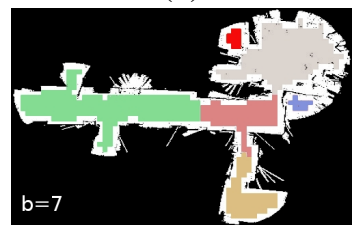

(d)
Figure 8: (a) Safely accessible area of metric map (in green) and (b)-(d) topological segmentation for different values of the bandwidth $b$ in the kernel space clearance estimation.

small volumes do not naturally alter the topology of space. However, when small volumes approach one another or reside close to bigger volumes they have a combined effect and space topology is altered (e.g. in Figure 9 (d),(f) wherein the upper right region is confined due to the presence of an obstacle close to the wall).

A particular setting of the $t_{s}, \sigma$ and $b$ parameters depends on the size characteristics of the robot, the desired granularity of the segmentation process as well as the desired level of space clearance.

In Figure 10, we show the results of segmentation under a fixed setting of parameters $\left(\sigma=15, t_{s}=\right.$

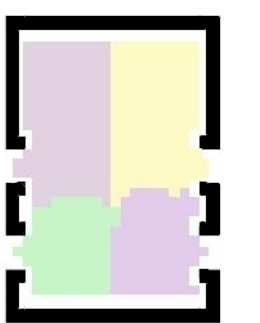

(a)

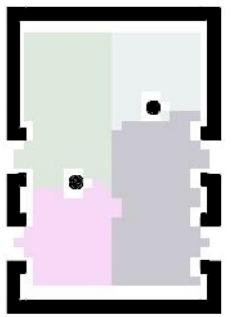

(d)

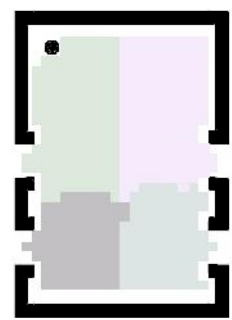

(b)

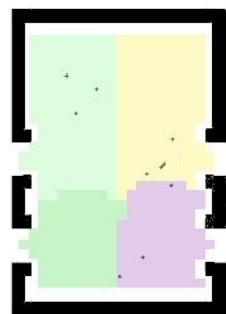

(e)

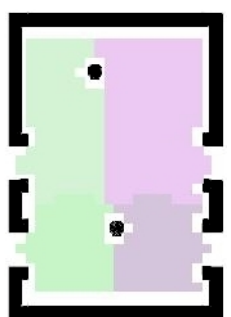

(c)

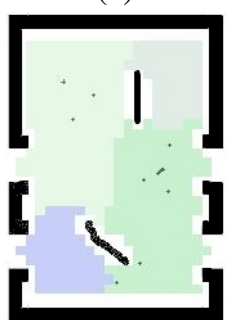

(f)
Figure 9: Topological clustering of free area in the presence of noise or relatively small obstacles; (a) initial clustering, (b)-(f) clustering under various arrangements of small obstacles or noise. 


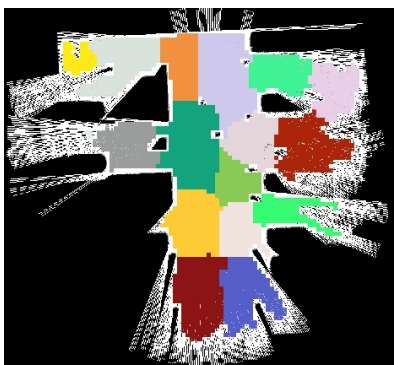

(a)

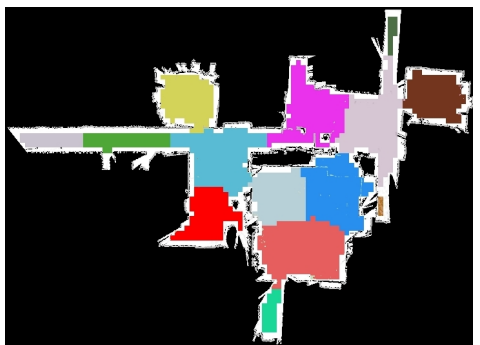

(d)

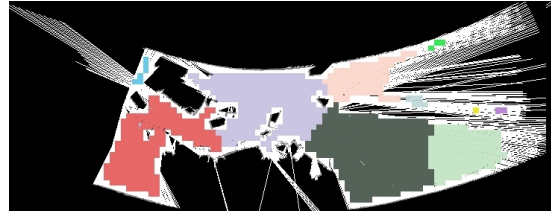

(b)

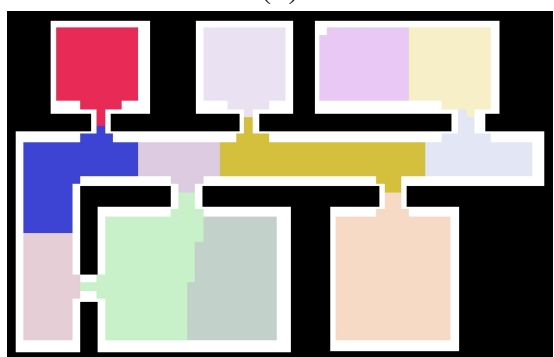

(e)

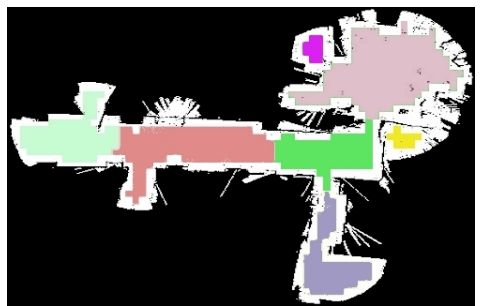

(c)

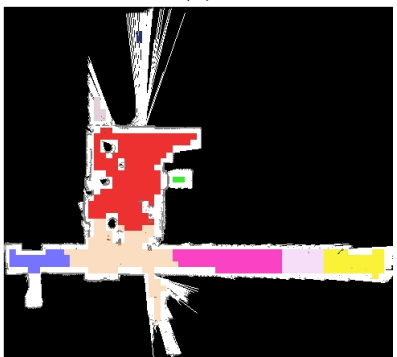

(f)

Figure 10: Topological segmentation of generic metric maps; (a) parking lot, (b) tunnel car accident, (c) warehouse, (d) office environment, (e) simulated indoor environment, (f) hallway.

$0.9, b=5)$ in a variety of metric maps ranging from structured to totally unstructured.

In the case of structured environments, the areas corresponding to medium sized rooms are identified as individual regions while larger constrained areas such as corridors or halls, are segmented to more than one region. Hence, by adopting this approach elongated areas are segmented. Alternatively, we could decrease the granularity of segmentation by increasing the $b$ parameter as shown in Figure 8. Another possibility would be to fuse distinct areas that belong to an elongated area by comparing the convexity of the fused regions against that of the resulting region.

In the case of unstructured maps where there is no notion of rooms or corridors, the only meaningful segmentation criterion corresponds to the identification of regions where space clearance is locally high as well as regions that are spatially disjoint-isolated and hence unreachable by the robot. As can be seen from the respective examples in Figure 8, these areas are well defined by the proposed segmentation method

The connectivity among the regions, i.e. the edges of the corresponding topological graph can be straightforwardly obtained by checking the $6^{\text {th }}-8^{\text {th }}$ neighbourhood of each cell.

The average segmentation time for the maps shown in Figure 10 using a Linux-based system equipped with an Intel Core i7 CPU 860 was 0.41 secs. The overall computational cost of the proposed algorithm is dominated by the mean-shift clustering stage as the preceding stage involving the computation of the space clearance can be efficiently computed by exploiting the separability property of the gaussian kernel. Furthermore, if necessary, the efficiency of the mapping process could be improved by clustering different cells in parallel.

\section{CONCLUSIONS AND FUTURE WORK}

We have presented a new topological mapping method that is robust to noisy sensory data and does not presume a particular structure of the environment. By an initial parameterization that is imposed by the characteristics of the robot, it can be readily applied to generic metric maps of minimal environmental constraints, as demonstrated in a variety of real-world examples. As a result of its formulation, the proposed approach can in parallel serve as a low level pathplanning algorithm by using the consecutive meanshift vectors of the convergence paths. This feature together with the real-time performance of the topological segmentation increases the overall efficiency of the robot by allowing for allocation of its computational resources to tasks other than path-planning.

As part of our future work, we are considering the application of the proposed method for 3D topological mapping as well as segmentation of generic $3 \mathrm{D}$ volumes, since the notion of $2 \mathrm{D}$ space clearance 
can be readily extended to 3D. In addition, it appears that the proposed algorithm could be improved by introducing appropriate criteria for fusing topological regions that are part of constrained elongated areas, whenever this is required.

\section{ACKNOWLEDGEMENT}

This paper describes research done under the EUFP7 ICT 247870 NIFTI project.

\section{REFERENCES}

Beeson, P., Jong, N., and Kuipers, B. (2005). Towards autonomous topological place detection using the extended voronoi graph. In IEEE ICRA.

Beeson, P., MacMahon, M., Modayil, J., Provost, J., Savelli, F., and Kuipers, B. (2003). Exploiting local perceptual models for topological map-building. In IJCAI Workshop on Reasoning with Uncertainty in Robotics.

Brunskill, E., Kollar, T., and Roy, N. (2007). Topological mapping using spectral clustering and classification. In IEEE IRS

Choi, J., Choi, M., and Chung, W. (2009). Incremental topological modeling using sonar gridmap in home environment. In IEEE IRS.

Comaniciu, D. and Meer, P. (2002). Mean shift: a robust approach toward feature space analysis. IEEE Transactions on Pattern Analysis and Machine Intelligence, 24(5):603-619.

Eppstein, E., Konolige, K., and Marthi, B. (2006). Hierarchical map building and planning based on graph partitioning. In IEEE ICRA.

Fabrizi, E. and Saffiotti, A. (2000). Extracting topologybased maps from gridmaps. In IEEE ICRA.

Fabrizi, E. and Saffiotti, A. (2002). Augmenting topologybased maps with geometric information. Robotics and Autonomous Systems, 40(2-3):91-97.

Fukunaga, K. and Hostetler, L. (1975). The estimation of the gradient of a density function, with applications in pattern recognition. IEEE Transactions on Information Theory, 21(1):32-40.

Gianni, M., Papadakis, P., Pirri, F., Liu, M., Pomerleau, F., Colas, F., Zimmermann, K., Svoboda, T., Petricek, T., Kruijff, G., Khambhaita, H., and Zender, H. (2011). A unified framework for planning and executionmonitoring of mobile robots. In AAAI Workshop on Automated Action Planning for Autonomous Mobile Robots.

Goedeme, T., Nuttin, M., Tuytelaars, T., and Gool, L. (2007). Omnidirectional vision based topological navigation. International Journal of Computer Vision, 74:219-236.

Goedeme, T., Tuytelaars, T., and Gool, L. . (2008). Visual topological map building in self-similar environments.
In Informatics in Control Automation and Robotics, volume 15, pages 195-205.

Joo, K., Lee, T., Baek, S., and Oh, S. (2010). Generating topological map from occupancy grid-map using virtual door detection. In IEEE Congress on Evolutionary Computation.

Kuipers, B., Modayil, J., Beeson, P., MacMahon, M., and Savelli, F. (2004). Local metrical and global topological maps in the hybrid spatial semantic hierarchy. In IEEE ICRA.

Liu, M., Colas, F., and Siegwart, R. (2011). Regional topological segmentation based on mutual information graphs. In IEEE ICRA.

Liu, M., Scaramuzza, D., Pradalier, C., Siegwart, R., and Qijun, C. (2009). Scene recognition with omnidirectional vision for topological map using lightweight adaptive descriptors. In IEEE IRS.

Maxwell, B., Smart, W., Jacoff, A., Casper, J., Weiss, B., Scholtz, J., Yanco, H., Micire, M., Stroupe, A., Stormont, D., and Lauwers, T. (2004). 2003 aaai robot competition and exhibition. AI Magazine, 25(2):6880.

Montijano, E. and Sagues, C. (2011). Distributed multicamera visual mapping using topological maps of planar regions. Pattern Recognition, 44(7):1528-1539.

Murillo, A., Abad, P., Guerrero, J., and Sagues, C. (2009). Improving topological maps for safer and robust navigation. In IEEE IRS.

Murillo, A., Sagues, C., Guerrero, J., Goedeme, T., Tuytelaars, T., and Gool, L. (2007). From omnidirectional images to hierarchical localization. Robotics and $\mathrm{Au}$ tonomous Systems, 55(5):372-382.

Murphy, R. (2004). Human-robot interaction in rescue robotics. IEEE Transactions on Systems, Man, and Cybernetics, Part C: Applications and Reviews, 34(2):138-153.

Myung, H., Jeon, H., Jeong, W., and Bang, S. (2009). Virtual door-based coverage path planning for mobile robot. In Advances in Robotics.

Paul, R. and Newman, P. (2010). Fab-map 3d: Topological mapping with spatial and visual appearance. In IEEE ICRA.

Shi, J. and Malik, J. (2000). Normalized cuts and image segmentation. IEEE Transactions on Pattern Analysis and Machine Intelligence, 22(8):888-905.

Theoharis, T., Papaioannou, G., Platis, N., and Patrikalakis, N. (2008). Graphics \& Visualization: Principles \& Algorithms. A K Peters.

Thrun, S. (1998). Learning metric-topological maps for indoor mobile robot navigation. Artificial Intelligence, 99(1):21-71.

Valgren, C. and Lilienthal, A. (2008). Incremental spectral clustering and seasons: Appearance-based localization in outdoor environments. In IEEE ICRA.

Zivkovic, Z., Bakker, B., and Krose, B. (2006). Hierarchical map building and planning based on graph partitioning. In IEEE ICRA. 